\title{
FPGA Based Digital Implementation of Adaptive Synchronization Methodology for 6-D Chaotic System
}

\author{
Rameshbabu Ramar ${ }^{1}$ and G R Suresh ${ }^{2}$ \\ ${ }^{1}$ Research Scholar, St. Peter's Institute of Higher Education and Research, India \\ ${ }^{2}$ Professor, St. Peter's Institute of Higher Education and Research, India \\ ${ }^{1}$ rrameshbabu15@gmail.com, ${ }^{2}$ gureshgr@rediffmail.com
}

\begin{abstract}
In this paper, we proposed the FPGA based digital implementation of synchronization methodology for 6-D chaotic systems via nonlinear feedback adaptive control technique. We derived new results for the adaptive controllers and the parameter update laws based on Lyapunov stability theory to achieve the synchronization between identical 6-D chaotic systems. Since the digitization of chaotic synchronization is necessary for digital communication, the proposed adaptive synchronization methodology is implemented in digital circuits based on Field Programmable Gate Array chip. The digital chaotic signal also generated using MATLAB simulink and Xilinx System Generator technology. The numerical simulation and FPGA outputs are used to prove the robustness and effectiveness of our proposed methodology.
\end{abstract}

Keywords: Chaotic system, Adaptive synchronization, FPGA implementation

\section{Introduction}

A dynamical system is called as chaotic system whenever its evolution sensitively depends on the initial conditions. Since the first chaotic system is proposed by Lorentz [1] for weather model, this topic has attracted the interest of many researchers and is still an important problem in the modern theory of chaotic synchronization. The chaos synchronization is the important phenomena for digital communication [2][3][4], digital modulation [5], digital under water communication [6], pseudorandom number generators [7][8], cryptography [9], digital image and video encryption [10][11][12] and many digital information systems. Since the digitalization of chaotic systems and its synchronization methodology is very important in digital information systems, the researchers focused on the FPGA based digital implementation of chaotic systems and its synchronization methodology [13][14][15][16][17] [18][19][20][21]. The researchers mostly prefers the Field Programmable Gate Array (FPGA) chips to generate digital chaotic signals since FPGA has high accuracy, short design circuit, low power consumption and low cost. In order to increase the chaos based application in engineering field, chaotic systems need flexible architecture support. With the digitalization and re-configurability of the FPGA, chaotic systems and their applications can be more flexible. Thus, different forms of signals can be easily generated with the change of parameters of chaotic systems.

Article history:

Received (February 25, 2020), Review Result (March 28, 2020), Accepted (May 4, 2020) 
The Lyapunov exponents represents that a chaotic system is complex and unpredictable in nature. The chaos synchronization is the process wherein two (or many) chaotic systems (either identical or non-identical) adjust a given property of their motion to a common behavior, due to coupling or forcing. This ranges from complete agreement of trajectories to locking of phases. In many of the synchronization methodology, drive - response or master slave configuration is used. In this configuration, a chaotic system called slave system is controlled by the output of another chaotic system called master system so that the trajectory of the slave system asymptotically approaches that of the master system and the error signal is zero. In last two decades many chaotic synchronization methodology are presented in literature review [22]-[39]. Compared to all other synchronization techniques, adaptive control method is simple and efficient to synchronize the chaotic systems. Based on the literature survey, the FPGA based digital implementation of adaptive synchronization methodology for 6-D chaotic system is proposed in this paper.

The rest of the paper is organized as follows: In section 2, a 6 dimensional chaotic system is described. In section 3, the detail of nonlinear feedback adaptive control method is studied. In section 4, non linear adaptive controllers are designed to synchronize the identical $6 \mathrm{D}$ chaotic systems. In section 5, FPGA based digital implementation of adaptive synchronization methodology for $6 \mathrm{D}$ chaotic system is proposed and MATLAB and Xilinx simulation results are given to prove the robustness and effectiveness of our proposed methodology. Finally the presented work is concluded in section 6 .

\section{Description of six - dimensional chaotic system}

The 6 - D chaotic system is given as follows,

$$
\begin{aligned}
\dot{x} & =-z-w \\
\dot{y} & =-r-b \\
\dot{z} & =x+\alpha z \\
\dot{r} & =y+\beta r \\
\dot{w} & =\gamma-\sigma w+x w-y b \\
\dot{b}= & -\delta b+y w+x b
\end{aligned}
$$

Here $\mathrm{x}, \mathrm{y}, \mathrm{z}, \mathrm{r}, \mathrm{w}$ and $\mathrm{b}$ are the state variables of 6-D chaotic system and the Equation 1, exhibits the chaotic behavior when the parameter values are,

$$
\alpha=\beta=\gamma=0.2, \sigma=\delta=5.7
$$

For the initial conditions $\{-2,1,5,3,-1,3.5\}$ and the parameter values as given above, the phase portraits are shown in [Figure 1].

\section{Theory of adaptive nonlinear feedback control methodology}

Consider the chaotic systems described by the dynamics,

$$
\dot{x_{1}}=\alpha x_{1}+f\left(x_{1}\right)
$$

Where $\mathrm{x}_{1} \in \mathrm{R}^{\mathrm{n}}$ are the states of the system, $\alpha$ is the $\mathrm{n} \mathrm{x}$ n matrix of the system parameters and $f: R^{n} \rightarrow R^{n}$ is the non-linear part of the system. We consider Equation 2, as the mater or drive system. As the slave or response system, we consider the following,

$$
\dot{x_{2}}=\beta x_{2}+g\left(x_{2}\right)+u(t)
$$


Where $\mathrm{x}_{2} \in \mathrm{R}^{\mathrm{n}}$ are the states of the system, $\beta$ is the $\mathrm{n} \mathrm{x}$ matrix of the system parameters and $\mathrm{g}: \mathrm{R}^{\mathrm{n}} \rightarrow \mathrm{R}^{\mathrm{n}}$ is the non-linear part of the system and $\mathrm{u} \in \mathrm{R}^{\mathrm{n}}$ are the adaptive controllers of the

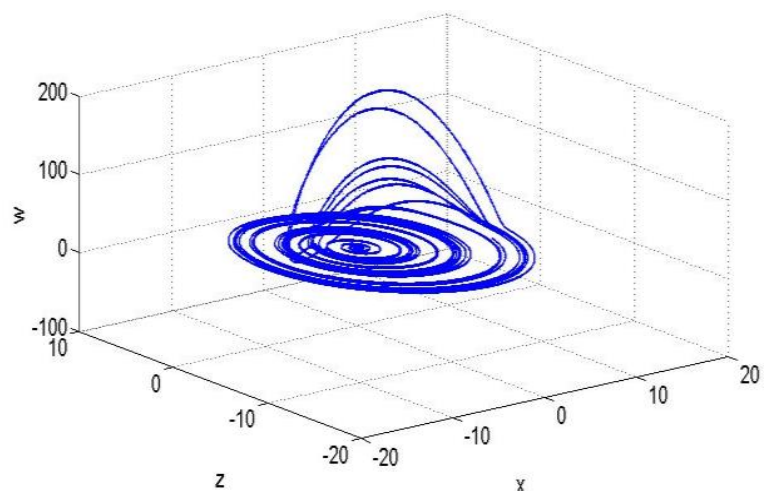

(a) $\mathrm{X}-\mathrm{Z}-\mathrm{W}$ plane

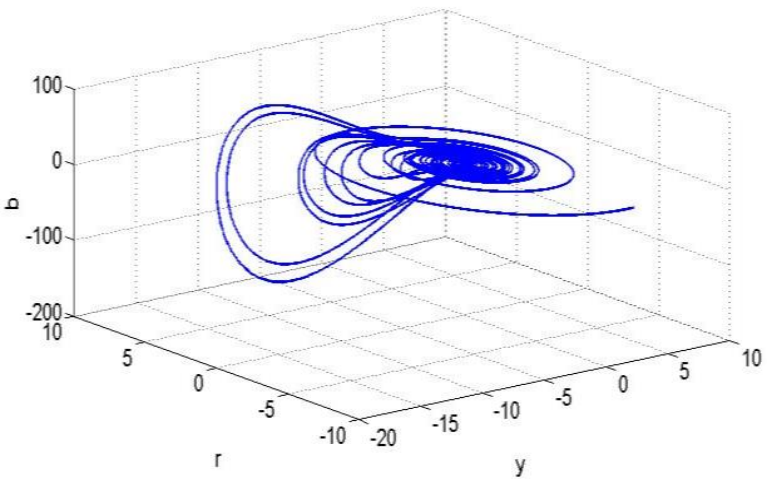

(b) y - r - b plane

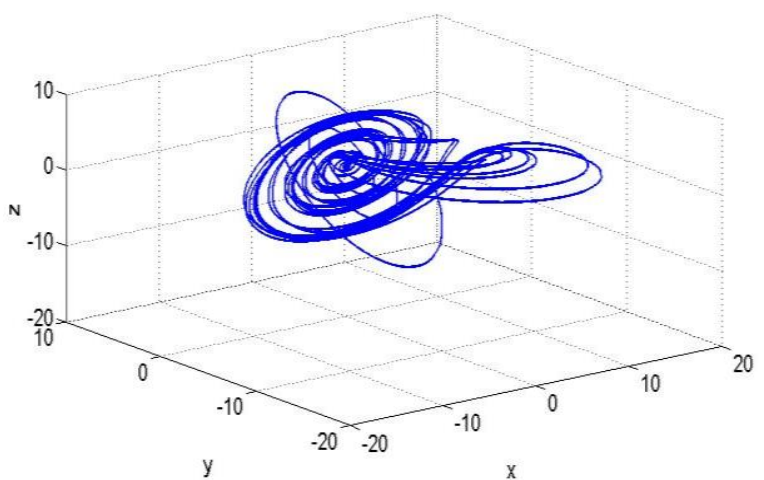

(c) $x-y-z$ plane

Figure 1. The Phase Portraits of Chaotic System (1) for the parameter values $\alpha=\beta=\gamma=0.2, \sigma=$ $\delta=5.7$ and the initial conditions $\{-2,1,5,3,-1,3.5\}$ 
slave system. If $\boldsymbol{\alpha}=\boldsymbol{\beta}$ and $\mathrm{f}=\mathrm{g}$ then $\mathbf{x}_{\mathbf{1}}$ and $\mathbf{x}_{2}$ represents the states of two identical chaotic systems otherwise $\mathbf{x}_{\mathbf{1}}$ and $\mathbf{x}_{\mathbf{2}}$ represents the states of two non-identical chaotic systems. We define the complete synchronization error as,

$$
e=x_{2}-x_{1}
$$

The synchronization error dynamics can be obtained as,

$$
\dot{e}=\beta x_{2}+g\left(x_{2}\right)-\alpha x_{1}-f\left(x_{1}\right)+u(t)
$$

For the synchronization of chaotic systems, the adaptive controller is taken as,

$$
u(t)=u\left(x_{1}, x_{2}, \hat{\alpha}, \hat{\beta}\right)
$$

Here $\hat{\alpha}$, and $\hat{\beta}$ is an estimate of the unknown parameter vector $\alpha$ and $\beta$ respectively. The synchronization problem is to find the controller $u$ to stabilize the error dynamics (4) for all initial conditions $\mathrm{e}(0) \in \mathrm{R}^{\mathrm{n}}$. Hence, we find a controller $\mathrm{u}$ so that $\lim \|e(t)\|=0$ for all $\mathrm{e}(0) \in \mathrm{R}^{\mathrm{n}}$. Consider a quadratic Lyapunov function defined as follows,

$$
V=\frac{1}{2}\left(e^{T} e+e_{\beta_{1}}^{2}+e_{\beta_{2}}^{2}+\cdots+e_{\beta_{k}}^{2}\right)
$$

Then the Lyapunov function $\mathrm{V}$ is differentiated along the trajectories of the error dynamics and parameter update law is carefully designed so that $\mathrm{V}$ is a negative definite function everywhere. This ensures that both the errors of synchronization between the outputs of the chaotic master and slave systems and the parameter estimation error are given by,

$e_{\beta}=\beta-\hat{\beta}$ decay to zero exponentially.

\section{Design of adaptive controllers for the synchronization of 6 - D chaotic system}

In this section, we investigate the complete synchronization between identical six dimensional chaotic systems based on the non-linear feedback adaptive control theory. Equation 6 represents the master or drive system as,

$$
\begin{gathered}
\dot{x_{1}}=-\left(z_{1}+w_{1}\right) \\
\dot{y_{1}}=-\left(r_{1}+b_{1}\right) \\
\dot{z_{1}}=x_{1}+\alpha z_{1} \\
\dot{r_{1}}=y_{1}+\beta r_{1} \\
\dot{w_{1}}=\gamma-\sigma w_{1}+x_{1} w_{1}-y_{1} b_{1} \\
\dot{b_{1}}=-\delta b_{1}+y_{1} w_{1}+x_{1} b_{1}
\end{gathered}
$$

Here, $x_{1}, y_{1}, z_{1}, r_{1}, w_{1}$ and $b_{1}$ are the state variables of master system, the system parameters are $\alpha=\beta=\gamma=0.2, \sigma=\delta=5.7$. The Equation 7 is considered as the controlled slave system,

$$
\begin{gathered}
\dot{x_{2}}=-\left(z_{2}+w_{2}\right)+u_{1} \quad \dot{y_{2}}=-\left(r_{2}+b_{2}\right)+u_{2} \\
\dot{z_{2}}=x_{2}+\alpha z_{2}+u_{3} \quad \dot{r_{2}}=y_{2}+\beta r_{2}+u_{4} \\
\dot{w_{2}}=\gamma-\sigma w_{2}+x_{2} w_{2}-y_{2} b_{2}+u_{5} \\
\dot{b_{2}}=-\delta b_{2}+y_{2} w_{2}+x_{2} b_{2}+u_{6}
\end{gathered}
$$




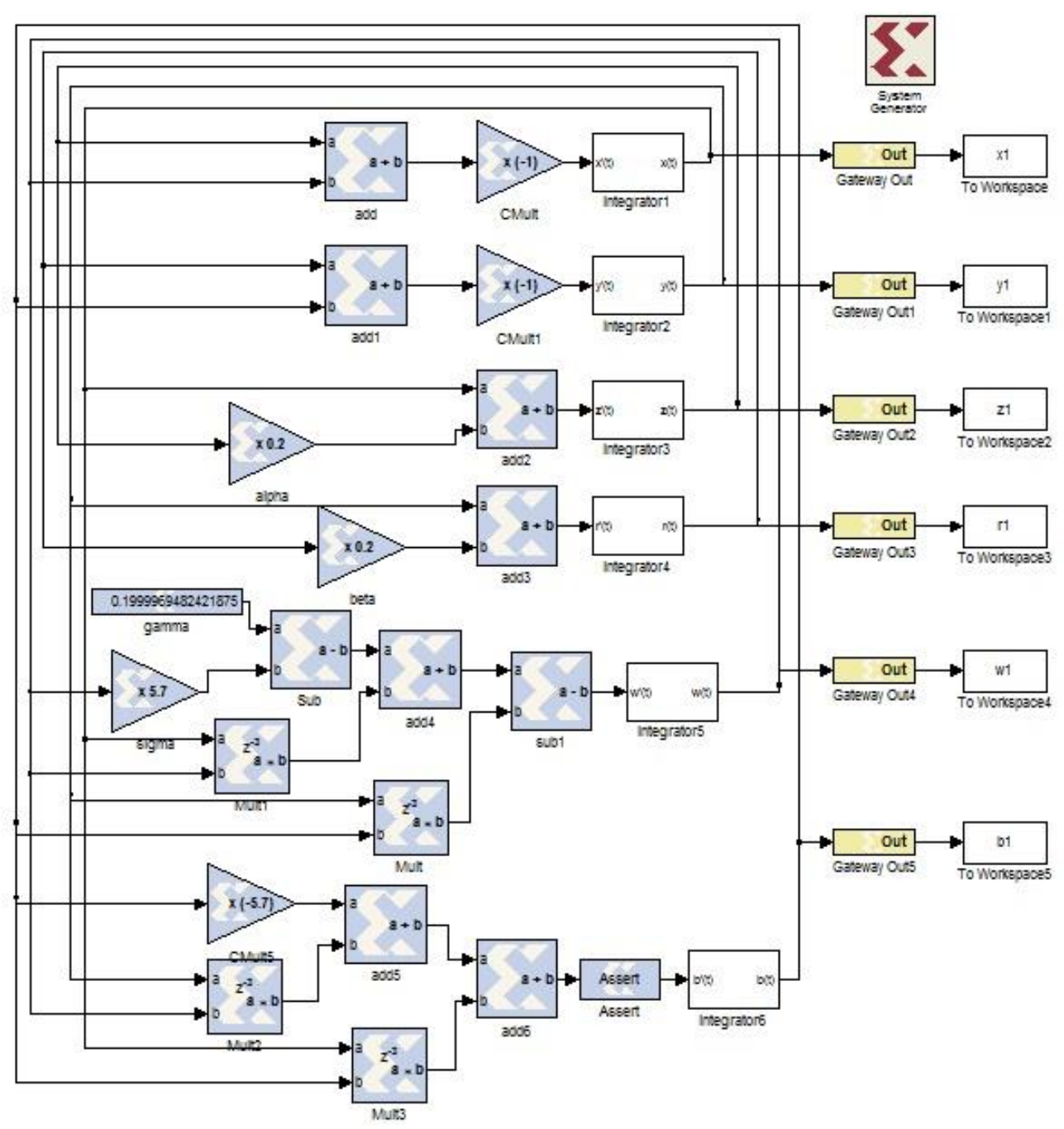

Figure 2. Construction of six dimensional chaotic system

In Equation $7, \boldsymbol{x}_{2}, \boldsymbol{y}_{2}, \boldsymbol{z}_{2}, \boldsymbol{r}_{\mathbf{2}}, \boldsymbol{w}_{\mathbf{2}}$ and $\boldsymbol{b}_{2}$ represents the state variables of slave system and $\boldsymbol{u}_{1}, \boldsymbol{u}_{2}, \boldsymbol{u}_{3}, \boldsymbol{u}_{4}, \boldsymbol{u}_{5}$ and $\boldsymbol{u}_{6}$ are the adaptive controllers used for the synchronization of master and slave chaotic system. The complete synchronization error dynamics are defined as,

$$
\begin{aligned}
& \dot{e}_{1}=\dot{x}_{2}-\dot{x}_{1}, \dot{e}_{2}=\dot{y}_{2}-\dot{y}_{1}, \dot{e}_{3}=\dot{z}_{2}-\dot{z}_{1}, \\
& \dot{e}_{4}=\dot{r}_{2}-\dot{r}_{1}, \dot{e}_{5}=\dot{w}_{2}-\dot{w}_{1}, \dot{e}_{6}=\dot{b}_{2}-\dot{b}_{1}
\end{aligned}
$$

The Controllers $U$ and the parameter update laws are derived for the adaptive control functions such that the error dynamics drives to zero,

$$
\begin{aligned}
& \mathrm{u}_{1}=\mathrm{e}_{3}+\mathrm{e}_{5}-\mathrm{e}_{1} \\
& \mathrm{u}_{2}=\mathrm{e}_{4}+\mathrm{e}_{6}-\mathrm{e}_{2} \\
& \mathrm{u}_{3}=-\mathrm{e}_{1}-\widehat{\alpha} \mathrm{e}_{3}-\mathrm{e}_{3}
\end{aligned}
$$




$$
\begin{gathered}
\mathrm{u}_{4}=-\mathrm{e}_{2}-\widehat{\beta} \mathrm{e}_{4}-\mathrm{e}_{4} \\
\mathrm{u}_{5}=\widehat{\sigma} \mathrm{e}_{5}-\mathrm{x}_{2} \mathrm{w}_{2}+\mathrm{x}_{1} \mathrm{w}_{1}+\mathrm{y}_{2} \mathrm{~b}_{2}-\mathrm{y}_{1} \mathrm{~b}_{1}-\mathrm{e}_{5} \\
\mathrm{u}_{6}=\widehat{\delta} \mathrm{e}_{6}-\mathrm{y}_{2} \mathrm{w}_{2}-\mathrm{x}_{2} \mathrm{~b}_{2}+\mathrm{y}_{1} \mathrm{w}_{1}+\mathrm{x}_{1} \mathrm{~b}_{1}-\mathrm{e}_{6}
\end{gathered}
$$

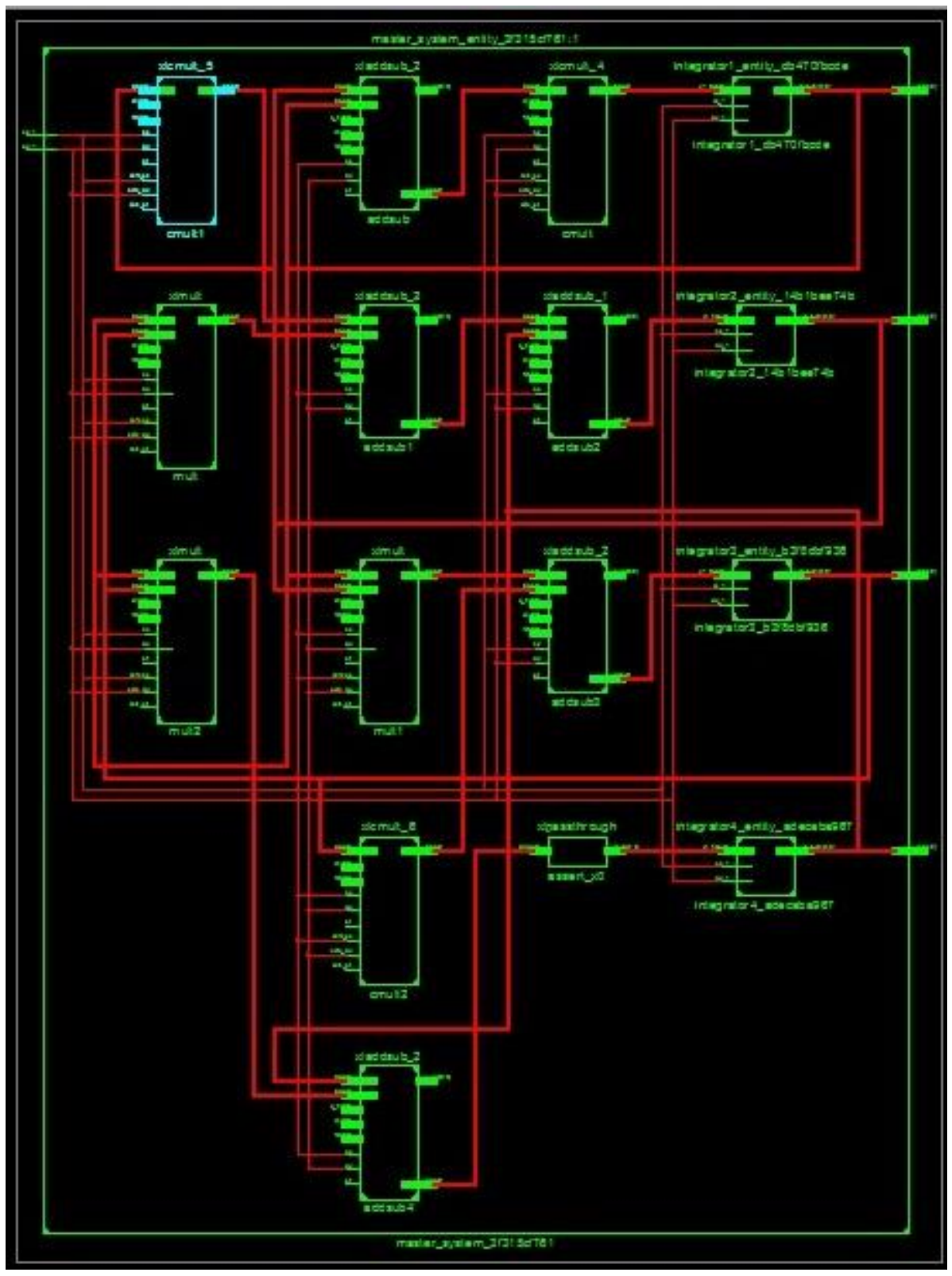

Figure 3. The RTL Schematic of 6 - D Chaotic System

And

$$
\dot{\hat{\alpha}}=\mathrm{e}_{3}^{2}, \dot{\hat{\beta}}=\mathrm{e}_{4}^{2}, \dot{\hat{\sigma}}=-\mathrm{e}_{5}^{2} \text { and } \dot{\hat{\delta}}=-\mathrm{e}_{6}^{2}
$$

Here $\hat{\alpha}, \hat{\beta}, \hat{\sigma}, \hat{\delta}$ are the estimate values of the unknown parameters $\alpha, \beta, \sigma$ and $\delta$ respectively. Consider a Lyapunov function candidate as, 


$$
\begin{gathered}
V=\frac{1}{2} e^{T} e+\frac{1}{2}\left(e_{\alpha}^{2}, e_{\beta}^{2}, e_{\sigma}^{2}, e_{\delta}^{2}\right) \\
\dot{V}=e_{1} \dot{e}_{1}+e_{2} \dot{e}_{2}+e_{3} \dot{e}_{3}+e_{4} \dot{e}_{4}+e_{5} \dot{e}_{5}+e_{6} \dot{e}_{6}+e_{\alpha} \dot{e}_{\alpha}+e_{\beta} \dot{e}_{\beta}+e_{\sigma} \dot{e}_{\sigma}+e_{\delta} \dot{e}_{\delta} \\
\dot{V}=-\left[e_{1}^{2}+e_{2}^{2}+e_{3}^{2}+e_{4}^{2}+e_{5}^{2}+e_{6}^{2}\right]
\end{gathered}
$$

The Equation 10 shows that the synchronization errors $e_{1}, e_{2}, e_{3}, e_{4}, e_{5}$ and $e_{6}$ and the parameter estimation error $e_{\alpha}, e_{\beta}, e_{\sigma}$ and $e_{\delta}$ decay to zero exponentially with time according to Lyapunov stability theory.

\section{FPGA based digital implementation of adaptive synchronization methodology for $6 \mathrm{D}$ chaotic system}

In this section, we implemented the proposed complete synchronization methodology in Field Programmable Gate Array (FPGA) Virtex4 xc4vfx100-12ff1152. The six dimensional chaotic system corresponding to [Equation 1], adaptive controllers corresponding to Equation 10 and parameter update laws corresponding to Equation 12 are constructed using MATLAB simulink and Xilinx system generator block sets.

[Figure 2] represents the implementation of 6-D chaotic system using Xilinx block set. We generated VHDL code from the system generator design. The generated VHDL code is simulated in Xilinx software and the RTL schematic diagram of 6 - D chaotic system is obtained as shown in [Figure 3]. [Figure 4] and 5 represents the implementation of adaptive controllers $u_{1}, u_{2}, u_{3}, u_{4}$ and $u_{5}, u_{6}$ using Xilinx block set respectively. Figure 6 represents the implementation of parameter update law in Xilinx block set. We adopt this implementation with a fixed point and with a representation of the real data on 32 bits (12Q20), 12 for the entire and 20 for the fraction.

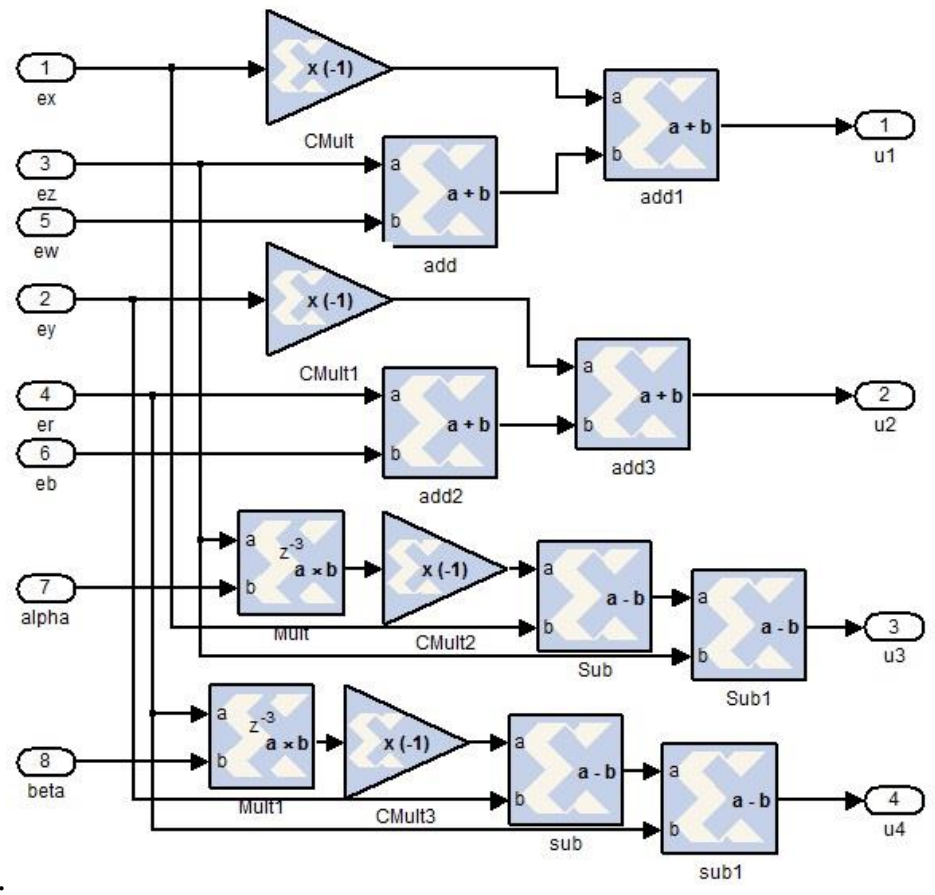

Figure 4. Design of adaptive controllers u1,u2,u3 and u4 


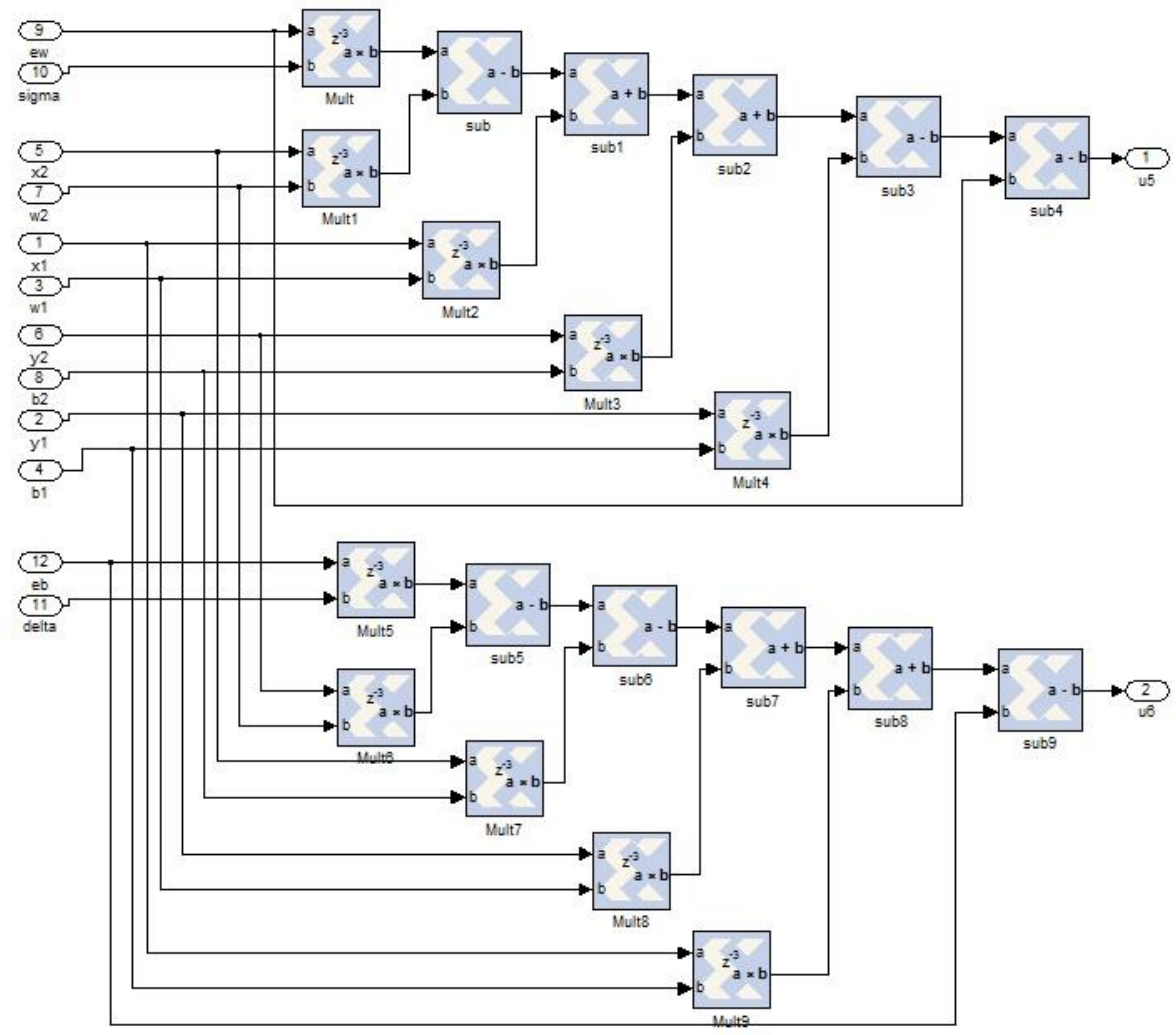

Figure 5. Design of adaptive controllers u5 and u6
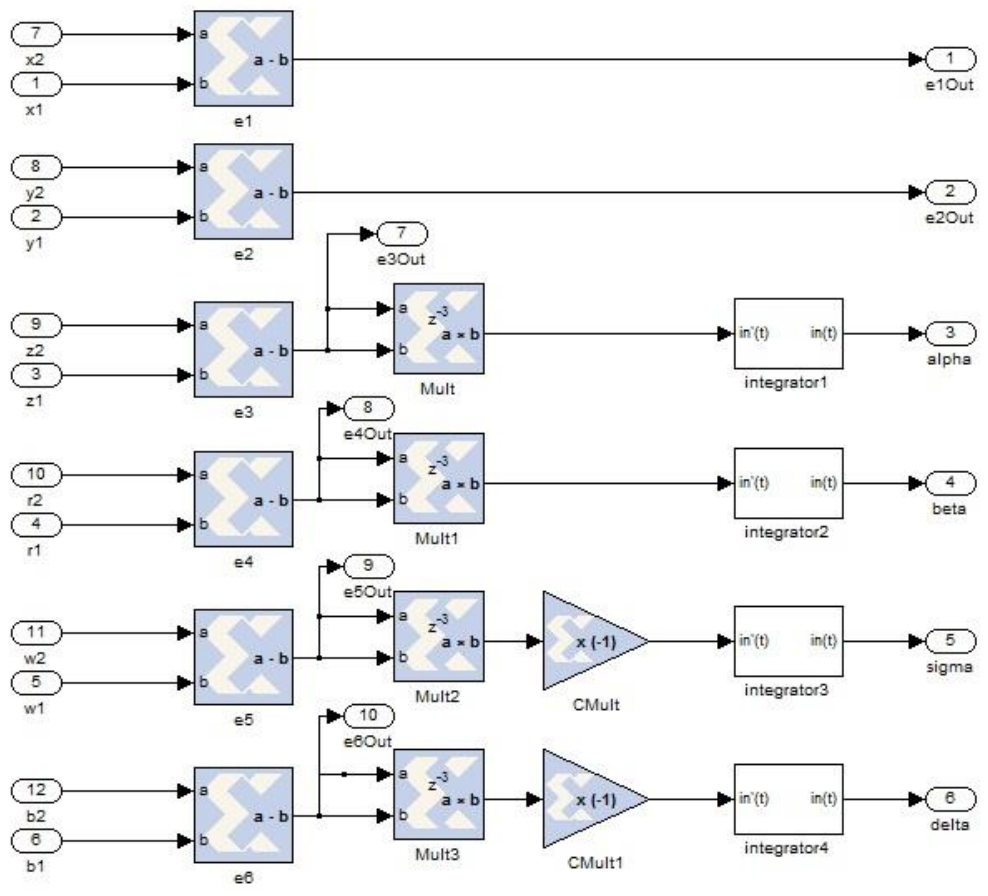

Figure 6. Design of parameter update law 
In numerical simulations, the initial conditions for master and slave 6-D chaotic systems are taken as $\{-2,1,5,3,-1,3,3.5\}$ and $\{8,8,-6,-1,3,1.5\}$ respectively. The initial values for unknown parameters $\alpha, \beta, \sigma$ and $\delta$ are respectively taken as $\{1,0.5,1.5,-2\}$. [Figure 7 (ab)] represents the synchronized state variables $x_{1}$ and $x_{2}, y_{1}$ and $y_{2}, z_{1}$ and $z_{2}, r_{1}$ and $r_{2}, w_{1}$ and $w_{2}$ and $b_{1}$ and $b_{2}$.

[Figure 8 (a)] represents the error signals $e_{1}, e_{2}, e_{3}, e_{4}, e_{5}, e_{6}$ that reaches the zero when the systems are synchronized together. [Figure 8(b)] represents the adaptive controller signals $u_{1}, u_{2}, u_{3}, u_{4}, u_{5}, u_{6}$ which become zero when the master and slave systems are synchronized together.

[Figure 9] represents the implementation of proposed synchronization methodology in MATLAB simulink Xilinx blockset.

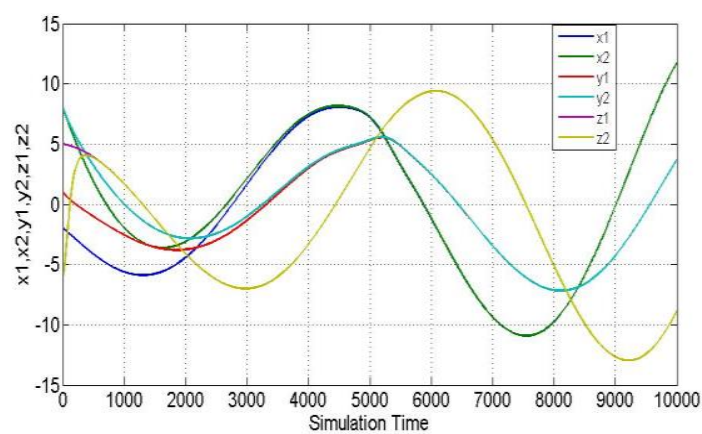

(a) $\mathrm{x} 1, \mathrm{x} 2, \mathrm{y} 1, \mathrm{y} 2, \mathrm{z} 1, \mathrm{z} 2$

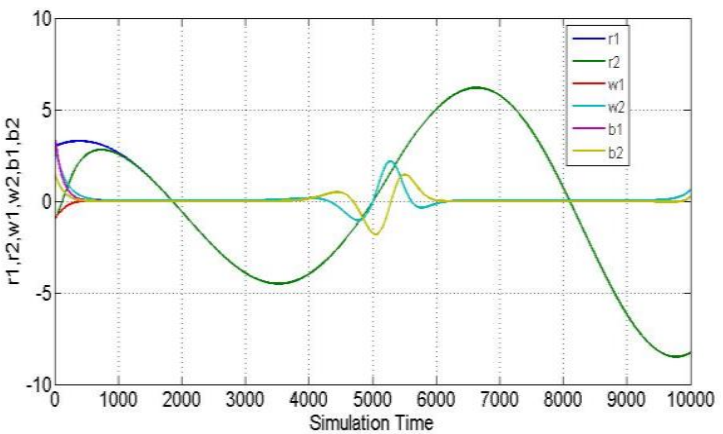

(b) $\mathrm{r} 1, \mathrm{r} 2, \mathrm{w} 1, \mathrm{w} 2, \mathrm{~b} 1, \mathrm{~b} 2$

Figure 7. Synchronized state variables

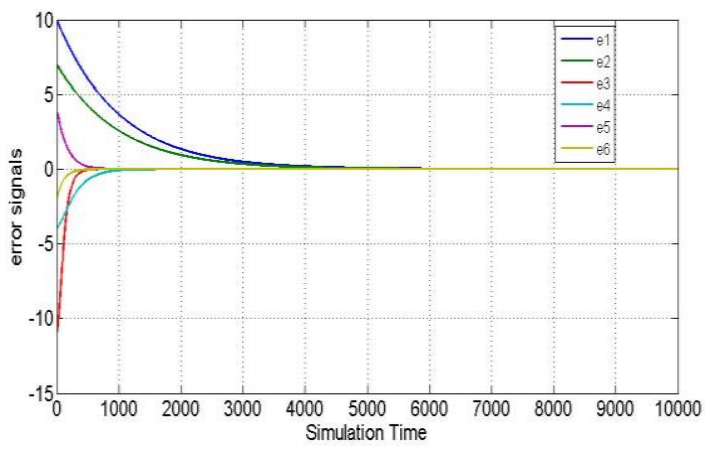

(a) e1, e2, e3, e4, e5 and e6

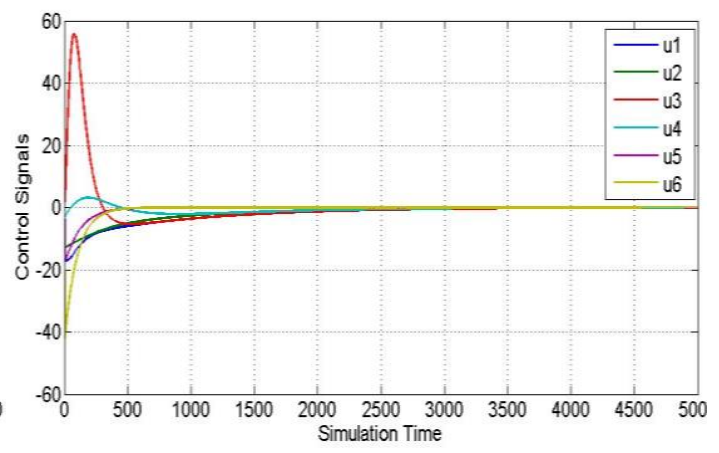

(b) $\mathrm{u} 1, \mathrm{u} 2, \mathrm{u} 3, \mathrm{u} 4, \mathrm{u} 5$ and $\mathrm{u} 6$

Figure 8. (a) Error signals and (b) Adaptive Controller signals

After that, VHDL code for completely synchronized $6 \mathrm{D}$ chaotic system is generated from the MATLAB simulink design shown in [Figure 9]. Then the VHDL code is simulated using Xilinx software and the source consumed by the synchronized 6 - D chaotic system is obtained as given in Table 1. Also RTL schematic diagram for synchronized 6 - D chaotic system is generated as shown in [Figure 10]. Note that the blocks and connections in RTL Schematic are corresponding to simulink design shown in [Figure 9]. 
The result of VHDL code simulation using Xilinx Isim simulator is shown in [Figure 11] which presents the portion of the waveform of synchronized signal $x_{1}$ and $x_{2}$. the signal $x_{1}$ has the numerical value -2 and the signal $x_{2}$ has the numerical value 8 .

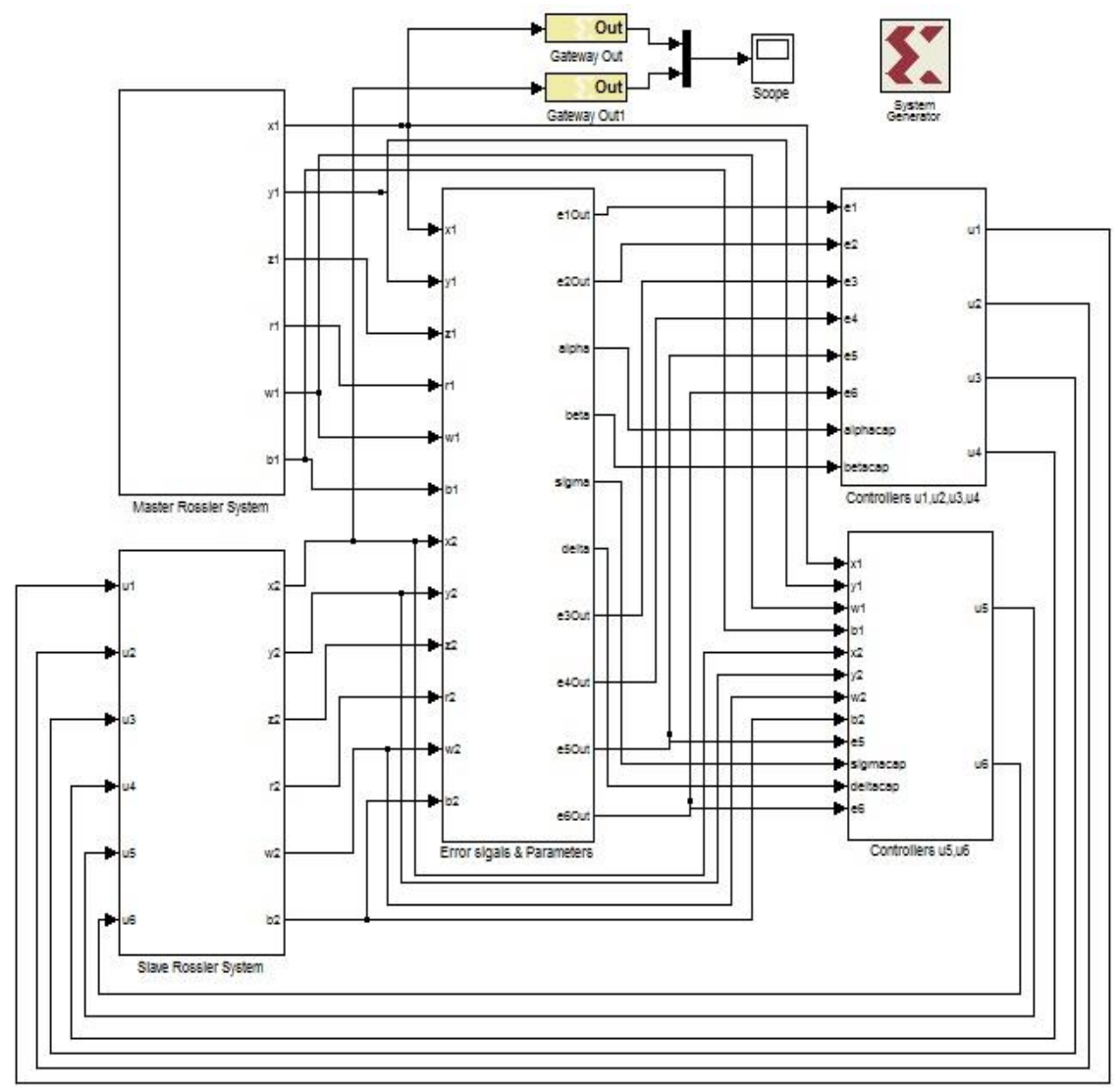

Figure 9. Implementation of synchronized 6 D system in Xilinx blockset

Table 1 . Source consumed by synchronized $6 \mathrm{D}$ chaotic system

\begin{tabular}{|c|c|c|}
\hline Logic Utilization & Used & Available \\
\hline No. of slice Flip Flops & 1,471 & 84,352 \\
\hline No. of 4 input LUT & 5,060 & 84,352 \\
\hline No. of occupied slices & 3,359 & 42,176 \\
\hline No. of slices containing only related logic & $3,3,59$ & 3,359 \\
\hline Total No. of 4 input LUTs & 5,983 & 84,352 \\
\hline
\end{tabular}




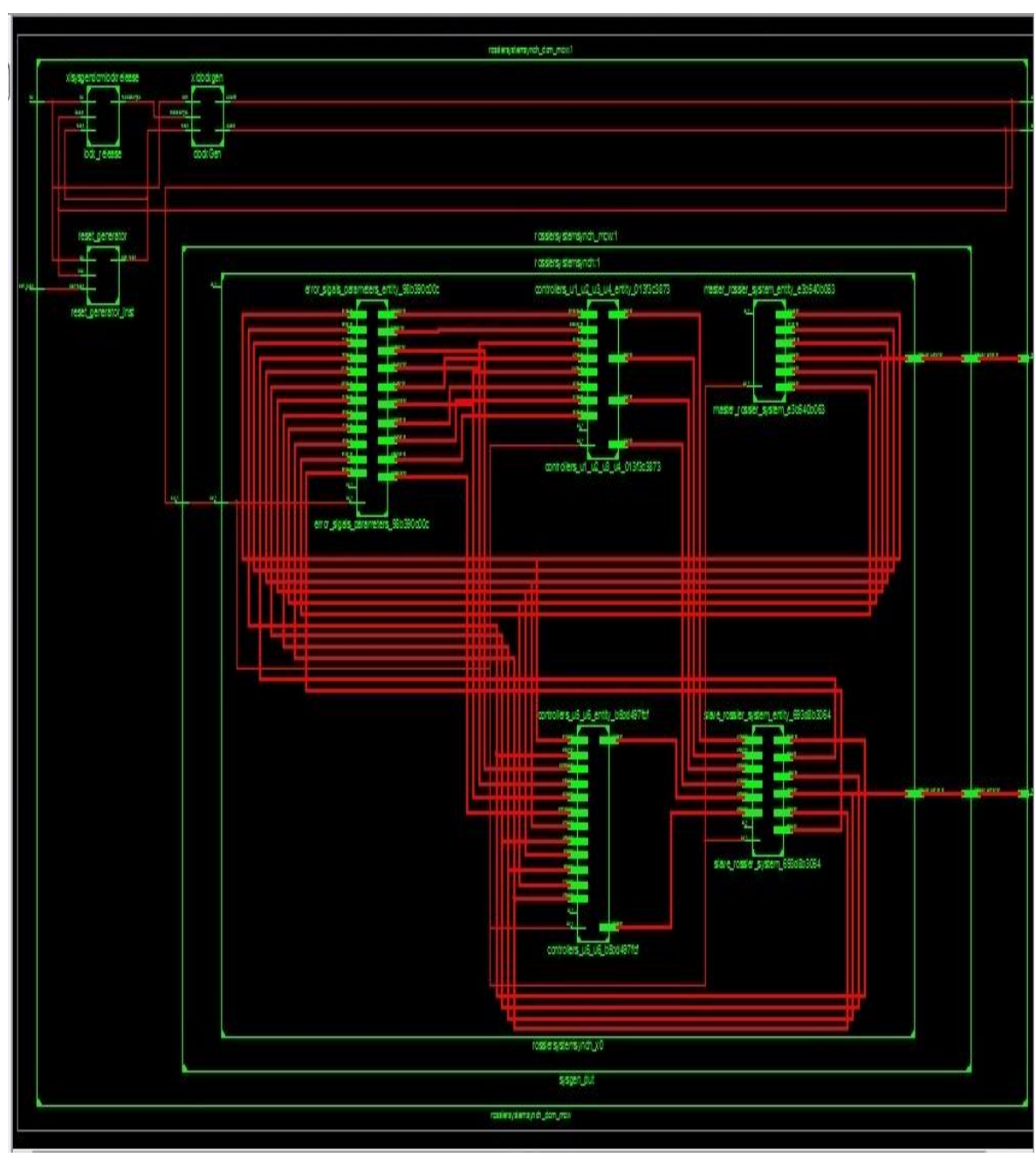

Figure 10. RTL schematic diagram of synchronized 6 D chaotic system

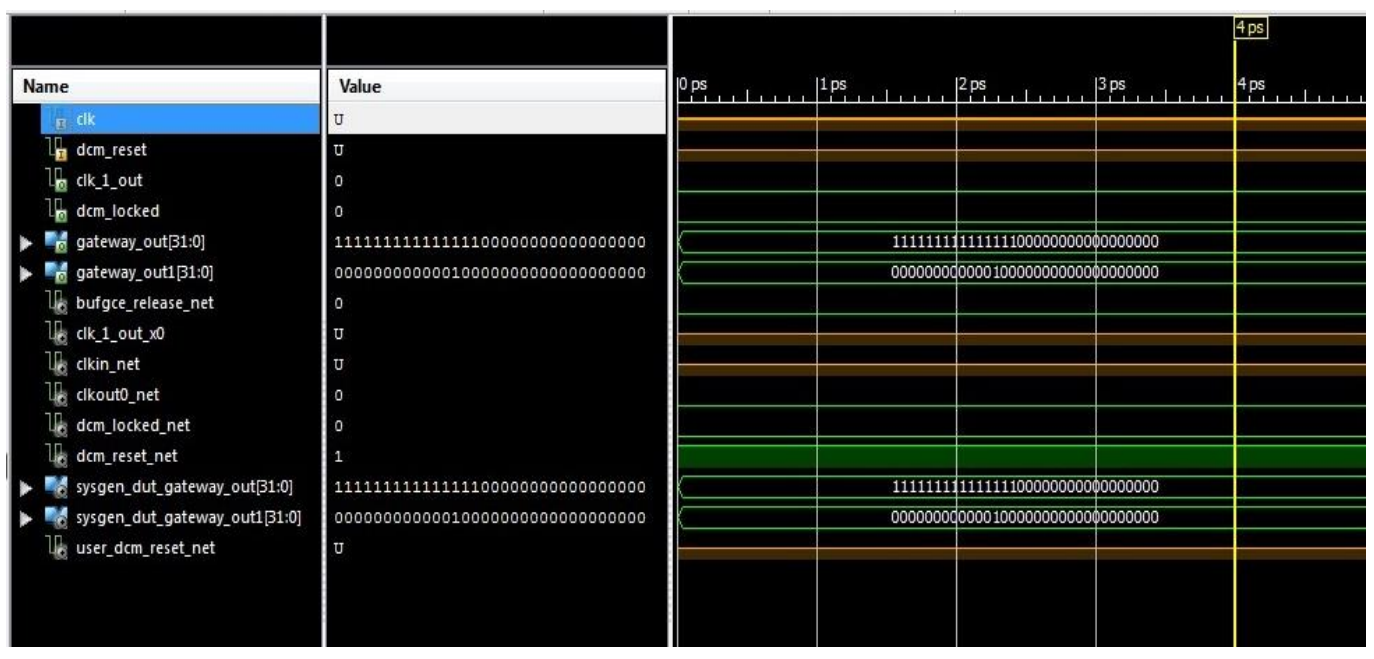

Figure 11. Xilinx simulation result 


\section{Conclusion}

In this paper, 6 dimensional chaotic system is introduced and adaptive synchronization methodology for $6 \mathrm{D}$ chaotic system is explained. We designed new adaptive controllers to achieve the complete synchronization of six dimensional chaotic systems. MATLAB simulation results are also given to prove the efficiency of proposed synchronization methodology. The proposed synchronization methodology is implemented in FPGA for the digitations of synchronized chaotic signals. The Xilinx simulation results are shown to validate and demonstrate the main results derived in this paper. The controller's performances were such that the complete synchronization of six dimensional chaotic system has been achieved. Xilinx System generators provide an efficient way to design a dynamic nonlinear control system using a FPGA board with hardware simulation. Therefore, reconfigurable devices provide very powerful tool for the control of complex nonlinear systems. The given six dimensional chaotic system consumes the source of FPGA most efficiently.

\section{References}

[1] E. N. Lorenz, "Deterministic non periodic flow" „Journal of Atmospheric Sciences, vol.20, pp.134, (1963) DOI: $10.1175 / 1520-0469$

[2] G. Kaddoum, "Wireless Chaos-Based Communication Systems: A Comprehensive Survey," IEEE Access, vol.4, pp.2621-2648, (2016) DOI:10.1109/ACCESS.2016.2572730

[3] G. A. Abib and M. Eisencraf, "On the performance of a digital chaos-based communication system in noisy channels," IFAC Papers online, vol.48, no.11, pp.976-981, (2015) DOI:10.1016/j.ifacol.2015.09.319

[4] N. X. Quyen, "On the study of quadrature DCSK modulation scheme for coginitive radio," International Journal of Bifurcation and Chaos, vol.27, no.09, 1750135, (2017) DOI: 10.1142/S0218127417501358

[5] X. Gao, M. F. Cheng, L. Deng, M. Zhang, S. Fu, and D. Liu, "Robust chaotic - shift - keying scheme based on electro optical hybrid feedback system," Optics Express, vol.28, no.08, pp.10847-10858, (2020) DOI: 10.1364/OE.389251

[6] C. Bai, H.P. Ren, M.S. Baptista, and C. Grebogi, "Digital underwater communication with chaos," Communications in Nonlinear Science and Numerical Simulation, vol.73, pp.14-24, (2019) DOI: 10.1016/j.cnsns.2019.01.02

[7] L. L. Bonilla, M. Alvaro, and M. Carretero, "Chaos based true random number generators," Journal of Mathematics in Industry, vol.7, no.1, (2016) DOI: 10.1186/s13362-016-0026-4

[8] M. Garcia-Bosque, A. Pérez-Resa, C. Sánchez-Azqueta, C. Aldea, and S. Celma, "Chaos-based bitwise dynamical pseudorandom number generator on FPGA," IEEE Transactions on Instrumentation and Measurement, vol.68, no.1, pp.291-293, ( 2019) DOI:10.1109/TIM.2018.2877859

[9] J. SenTeh, M. Alawida, and Y. Cheng Sii, "Implementation and practical problems of chaos based cryptography revisited," Journal of Information Security and Applications, vol.50, (2019) DOI: 10.1016/j.jisa.2019.102421

[10] Y. Luo, J. Yu, W. Lai, and L. Liu, “ A novel chaotic image encryption algorithm based on improved baker map and logistic map," Multimedia Tools and Applications, vol.78, pp.22023-22043, (2019) DOI: $10.1007 / \mathrm{s} 11042-019-7453-3$

[11] Z. Hua, Y. Zhou, and H. Huang, "Cosine transform based chaotic system for image encryption," Information Sciences, vol.480, pp.403-419 (2019) DOI: 10.1016/j.ins.2018.12.048

[12] Salama, Wessam, Elkamchouchi, Hassan, Abouelseoud, and Yasmine, "New Video Encryption Schemes Based on Chaotic Maps,” IET Image Processing, vol.14, no.2, (2020) DOI: 10.1049/iet-ipr.2018.5250.

[13] A.Ahmadi, K. Rajagopal, F.E. Alsaadi,V.T. Pham, F.E. Alsaadi, and S. Jafari, "A novel 5D chaotic system with extreme multi - stability and a line of equilibrium and its engineering applications: circuit design and 
FPGA implementation," Iranian Journal of Science and Technology, Transaction of Electrical Engineering, vol.44, pp.59-67, (2020) DOI: 10.1007/s40998-019-00223-5

[14] F. Yu, L. Liu, Binyong, Y. Huang, C. Shi, S. Cai, Y.Song, S. Du, and Q. Wan, "Analysis and FPGA realization of novel 5D hyperchaotic four wing memristor system, Active control synchronization and secure communication application," Complexity, vol.2019, Article ID 4047957, (2019) DOI: 10.1155/2019/4047957

[15] W.G. Yi, B.X. ei and W.Z. Lin, "Design and FPGA implementation of new hyperchaotic system," Chinese Physics B, vol.17, no.10 (2008) DOI: 10.1088/1674-1056/17/10/011

[16] I. Koyuncu, A.T. Özcerit, and I. Pehlivan, "Implementation of FPGA based real time novel chaotic oscillator," Nonlinear Dynamics, vol.77, pp.49-9, (2014) DOI: 10.1007/s11071-014-1272-x

[17] Z. Hua, B. Zhou, and Y. Zhou, "Sine-transform-based chaotic system With FPGA implementation," in IEEE Transactions on Industrial Electronics, vol.65, no.3, pp.2557-2566, (2018) DOI: 10.1109/TIE.2017.2736515

[18] M. Qiu, S.Yu, Y. Wen, J. Lü, J. He, andZ. Lin, "Design and FPGA implementation of a universal chaotic signal generator based on the verilog HDL fixed point algorithm and state machine control," International Journal of Bifurcation and Chaos, vol.27, no.03, (2017) DOI: 10.1142/S0218127417500407

[19] M. Lahcene, A. Adda, S. Naima Hadj, and M. Mustafa, "Design and FPGA implementation of Lorenz chaotic system for information security systems," Applied Mathematical Sciences, vol.7, pp.237-246, (2013) DOI: 10.12988/ams.2013.13022

[20] R. Rameshbabu and R. Karthikeyan, "Adaptive synchronization of novel chaotic system and its FPGA implementation," IEEE Int.conf. on Smart Technologies and Management for Computing, Communication, Controls, Energy and Materials, pp.517-522, (2015) DOI: 10.1109/icstm.2015.7225459

[21] R. Karthikeyan, A. Prasina, Ramesh Babu, S. Raghavendran, “ Indian Journal of Science and Technology, vol.8, no.11, pp.47901, (2015) DOI: 10.17485/ijst/2015/v8i11/71775

[22] J. Humberto Pérez-Cruz, Pedro A. Tamayo-Meza, Maricela Figueroa, Ramón Silva-Ortigoza, Mario PonceSilva, R. Rivera-Blas, and Mario Aldape-Pérez, "Exponential synchronization of chaotic Xian system using linear feedback control," Complexity, vol.2019, Article ID 4706491, (2019) DOI: 10.1155/2019/4706491

[23] F. Hannachi, "Analysis, dynamics and adaptive control synchronization of a novel chaotic 3-D system," SN Applied Sciences,” vol.1, no.158, (2019) DOI: 10.1007/s42452-019-0175-3

[24] A. Khan, M. Budhraja, and A. Ibraheem, "Synchronization among different switches of four non identical chaotic system via adaptive control," Arabian Journal for Science and Engineering, vol.44, pp.2717-2728 (2019) DOI: 10.1007/s13369-018-3458-x

[25] S. Vaidyanathan, O. Abba, B. Gambo, and M. Alidou, "A new three dimensional chaotic system: its adaptive control and circuit design,” International Journal of Automation and Control, vol.13, no.1, pp.101 - 121 (2019) DOI: 10.1504/ijaac.2019.10016561

[26] L. Kocarev and U. Partliz, "General Approach for Chaotic Synchronization with Applications to Communications,” Physical Review Letters vol.74, pp.5028-5030, (1995) DOI: 10.1103/physrevlett.74.5028

[27] J. $\mathrm{Lu}$ and X. Wu, "Synchronization of a Unified Chaotic System and the Application in Secure Communication,” Physics Letter A Vol. 305, pp.365 (2002) DOI: 10.1016/s0375-9601(02)01497-4

[28] E. Ott, C. Grebogi, and J.A Yorke, “Controlling chaos,” Phys. Review Letters, vol.64, pp.1196-1199, (1990) DOI: /10.1103/physrevlett.64.1196

[29] Ho and Hung, "Synchronization of two different chaotic systems using generalized active control," Phys Rev Letter A. vol.301, pp.424-8, (2002) DOI: 10.1515/ijnsns.2005.6.3.249

[30] M.T. Yassen, "Chaos synchronization between two different chaotic systems using active control," Chaos, Solitons Fractals, Vol. 23, pp.131-140, (2005) DOI: 10.1016/j.chaos.2004.03.038

[31] V. Sundarapandian and R. Karthikeyan, "Active controller design for global chaos anti synchronization of Li and Tigan chaotic systems," International Journal of Information Technology and Computer Science, vol.3, pp.255-268, (2011) DOI: 10.5121/ijcsit.2011.3420 
[32] T. Hamed, "On complete control and synchronization of Zhang chaotic system with uncertain parameters using adaptive control method," Nonlinear Engineering, vol.2017, pp.7, pp.45-50, (2017) DOI: 10.1515/nleng-2017-0050

[33] K. Ajith, K. Vijay, and Subir Das, "Synchronization of Time delay chaotic systems with uncertainties and external disturbances," Discontinuity, Nonlinearity, and Complexity, vol.8, pp.13-21, (2019) DOI: 10.5890/dnc.2019.03.002

[34] F.F. Cun, R.T. Yan, H.W. Ying, and Y.Y. Hai, "Active backstepping control of projective synchronization among different non-linear systems," Journal of Control, Measurements, Electronics, computing and communications, vol.58, pp.295-301, (2019) DOI: 10.1080/00051144.2018.1432466

[35] S.F. Jiunn, H.T. Jason Sheng, J.Y. Jun, and M.G. Shu, "Adaptive chattering free sliding mode control of chaotic systems with unknown input nonlinearity via smooth hyperbolic tangent function," Mathematical Problems in Engineering, vol.2019, pp.9, (2019) DOI: 10.1155/2019/4509674

[36] E. Deniz, P. Tiago, and S.W. Jereon, "Synchronization of chaos and its applications," Contemporary Physics, vol.58, pp.207 - 243, (2017) DOI: 10.1080/00107514.2017.1345844

[37] L. Chen, C. Huang, H. Liu, and Y. Xia, "Anti synchronization of a class of chaotic systems with applications to Lorenz system: A unified analysis of integer order and Fractional order," Mathematics, vol.7, pp.559, (2019) DOI: 10.3390/math7060559

[38] X. Chai and Z. Huigan, "Function projective lag synchronization of chaotic systems with certain parameters via adaptive - impulsive control," International Journal of Automation and computing, vol.16, pp.238-247, (2019) DOI: $10.1007 / \mathrm{s} 11633-016-1020-4$

[39] S. Vaidyanathan, A. Sambas, S. Kacar, and U. Cavusoglu, "A new finance chaotic system, its electronic realization, passivity based synchronization, and an application to voice encryption," Nonlinear Engineering, vol.8, pp.193 - 205, (2019) DOI:10.1515/nleng-2018-0012

\section{Authors}

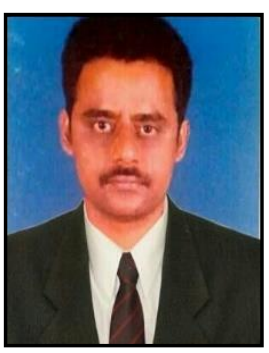

\section{R. Rameshbabu}

He completed M. Tech from Dr. M.G.R Deemed University, Chennai. He is pursuing doctorate at St. Peter's Institute of Higher Education and Research, Chennai. His areas of interests are chaotic system, synchronization of chaotic system and FPGA implementation.

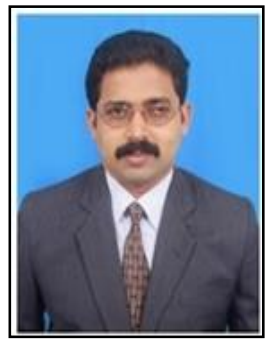

\section{G. R. Suresh}

$\mathrm{He}$ is working as professor in the Dept. of Biomedical engineering at St.Peter's Institute of Higher Education and Research, Chennai. He has published various research articles in referred International Journals and Conferences along with his scholars: more than 100 research articles indexed in Google Scholar with the hindex of 12, 63 articles indexed in Scopus and 38 research articles indexed in web of Science. 